INVESTIGACIÓN

http://dx.doi.org/10.15198/seeci.2017.43.29-51

Recibido: 14/03/2017 --- Aceptado: 27/04/2017

\title{
POTENCIAL PEDAGÓGICO DEL MOBILE LEARNING EN EL AULA DE MÚSICA EN SECUNDARIA
}

\section{METHODOLOGICAL POTENTIAL OF MOBILE LEARNING IN THE MUSIC CLASSROOM IN SECONDARY EDUCATION.}

Ma Encarnación Alises Camacho $^{\mathbf{1}}$ : Universidad Nacional a Distancia. España. encarniidem@gmail.com

\section{RESUMEN}

El presente estudio, basado en el proyecto musical nacido en el Centro de Formación del Profesorado de Castilla La Mancha en 2013, Crea la Banda Sonora de tu vida, pretende demostrar cómo el uso de dispositivos móviles en el aula de música en Secundaria, tiene un enorme potencial pedagógico y educativo, tomando como referencia las actuales metodologías educativas, como son las inteligencias múltiples, la inteligencia emocional, el trabajo colaborativo o el Mobile Learning, desde el aprendizaje por proyectos, el emprendimiento o la integración del modelo TPACK, desde una perspectiva relacional, proponiendo un modelo de enseñanza-aprendizaje que utilice adecuadamente el Mobile Learning.

El uso generalizado en su vida cotidiana de dispositivos móviles en la población estudiada, alumnado de Secundaria, nos obliga en cierta medida a incorporarlos a la escuela, con una base metodológica adecuada, relacionando educación formal, informal y no formal, utilizando el Mobile Learning como una estrategia de aprendizaje, adscrita a la competencia de aprender a aprender y a las demás competencias del s. XXI.

PALABRAS CLAVE: música - Mobile Learning - inteligencia emocional - modelo TPACK

\section{ABSTRACT}

The current study, based on the musical project born in the Regional Training Teaching Center in Castilla La Mancha in 2013, "Create the Soundtrack of Your Life", intends to prove how the usage of mobile devices in the music classroom in Secondary School has an enormous pedagogical and educative potential, taking as a

\footnotetext{
${ }^{1}$ Ma Encarnación Alises Camacho: Doctoranda en Educación en Entornos Digitales. UNED. Máster en Educación y Comunicación en Red. UNED. Máster en Redes sociales y Aprendizaje Digital. UNED. Maestra en Educación Musical en la Junta de Castilla La Mancha.

Correo: encarniidem@gmail.com
} 
reference the current educational methodologies, such as multiple intelligences, emotional intelligence, collaborative work or Mobile Learning, using learning based on projects, entrepreneurship or the integration of the TPACK model, from a relational point of view, proposing a teaching-learning model which uses Mobile Learning appropriately. The widespread usage of mobile devices in the studied population's daily life, that is, students from secondary education, compel us, to some extent, to incorporate these devices into the school, with a suitable methodological basis, relating formal, informal and non-formal education, using Mobile Learning as a learning strategy, assigned to the learning to learn competence, as well as the other competences in the 21st century.

KEY WORDS: music - Mobile Learning - emotional intelligence - TPACK model

\section{Cómo citar este artículo}

Alises Camacho, Ma E. (2017). Potencial pedagógico del Mobile Learning en el aula de música en secundaria [Methodological potential of mobile learning in the music classroom in secondary education] Revista de comunicación de la SEECI, 43, 29-51. Doi:http://dx.doi.org/10.15198/seeci.2017.43.29-51 Recuperado de http://www.seeci.net/revista/index.php/seeci/article/view/459

\section{INTRODUCCIÓN}

En la actualidad nos enfrentamos a dos problemáticas, bien diferenciadas, respecto al tema que nos ocupa. Por un lado, el currículo de música en Secundaria, que en la actual ley educativa de España, la LOMCE, es una asignatura optativa dentro del bloque de asignaturas específicas que debe elegir cada administración educativa o el propio centro educativo, que menoscaba la importancia de la música como lenguaje universal y medio de comunicación no verbal, que tiene en la vida diaria del ser humano un valor incuestionable e indiscutible. Por tanto, es una realidad, que la asignatura de música, en nuestro actual sistema educativo, ha sido desplazada del lugar que debería de tener en la educación del alumnado de Secundaria.

Y por otro lado, la utilización de dispositivos móviles, que maneja prácticamente todo el alumnado de esta etapa; en 2015, un $98 \%$ de los jóvenes de 10 a 14 años contaba ya con un teléfono de última generación con conexión a Internet (Ditrendia, 2016). El uso de dispositivos móviles no se está adaptando adecuadamente a la escuela, desarrollando todo el potencial pedagógico e instrumental que tienen, centrándose su uso, como advierte Herrington (2009), en un modelo instruccionaltransmisivo, en el que el profesor introduce contenidos y el alumnado accede a ellos a través de sus dispositivos móviles, es decir, que su uso se ha limitado a una interacción demasiado guiada, a una consulta de datos o a una simple organización administrativa.

Estos múltiples modos de comunicación, junto a la combinación de medios que proporcionan los dispositivos móviles, como son mezclas de sonidos, imágenes, 
textos, gesto y vídeos, abre un abanico de posibilidades perceptivas y expresivas que desafían a la escuela y, en especial, a la asignatura de música en Secundaria.

Las transformaciones tecnológicas han producido, pues, una brecha generacional que repercute en que los jóvenes estén "en otra frecuencia de onda perceptiva, mental y actitudinal" (Ferrés, 2000), que produce una disgregación entre dos estratos sociales: "los jóvenes actuales aparecen como los más indicados para poder comprender y usar las nuevas tecnologías que han ido poblando el mundo, lo que conlleva una fuerte diferenciación del mundo adulto, que nos impulsa en muchos casos a analizar este proceso como un proceso de cambio generacional producido en las sociedades (post)modernas... (Marta \& Gabelas, 2013, p. 15).

Con este cambio generacional, manejamos dos conceptos, el de computación (los dispositivos móviles son pequeñas computadoras) y el de comunicación, con una relación suficientemente fuerte con la educación como para tener, por lo menos en consideración estos dispositivos. El de computación se basa en el desarrollo de un lenguaje entre el hombre, la máquina y de ésta con otras máquinas. Al hablar de dispositivos móviles en el aula estamos hablando de comunicación. El uso de éstos en Crea Banda Sonora tiene tres funciones principales: como comunicación entre alumnado, como comunicación entre alumnado y el mundo exterior a éste y como facilitador tecnológico de la interpretación musical en directo; por tanto, la música sirve, una vez más, para comunicar desde un lenguaje emocional. Está clara la utilidad de los dispositivos móviles por tanto.

Lo que nos enseña Kodaly, es que la educación debe partir de aquello que el alumnado conoce, y desde ahí, y con la función de guía del profesor, que éste llegue al conocimiento que más le interese. El aprendizaje sólo se produce si se tiene curiosidad y si produce emoción, como demostró Gordon $\mathrm{H}$. Bower, el impacto de la emoción en nuestra memoria es inmenso. Si partimos de una enseñanza contextual, emocional y comunicativa tendremos la respuesta al por qué utilizar dispositivos móviles en el aula, que iremos desgranando paso a paso a lo largo del artículo.

Todo ello da sentido a la realización de este estudio sobre el modelo pedagógico que sustenta a los procesos de enseñanza-aprendizaje en el proyecto Crea la banda sonora de tu vida, proyecto que nació en el Centro de Formación del Profesorado de Castilla La Mancha, que en su primer año fue secundado por 20 profesores de la región y en un segundo año, por 58 profesores de Secundaria y Primaria y que ya está siendo adoptado y adaptado por profesorado de toda España, por su alto valor pedagógico y metodológico, intentando devolver el puesto que se merece a la asignatura de música dentro del currículo oficial. Un proyecto que pretende formar una orquesta en cada aula, autogestionada por el alumnado, que asume distintos roles dentro de ella y cuyo fin último es crear la banda sonora de su vida, que presenta en un concierto final, junto al resto del alumnado implicado en éste.

El proyecto tiene un enorme potencial pedagógico, que se vertebra desde seis pilares pedagógicos, que pasamos a explicar a continuación, añadiendo el aspecto relacional 
de los dispositivos móviles. Con estos seis pilares pedagógicos se irá desgranando con mayor amplitud en qué consiste.

\subsection{Inteligencia emocional}

La inteligencia emocional está totalmente presente en el proyecto Crea la banda sonora de tu vida y no podemos dejar de contemplarla en nuestra investigación, por la importancia capital que tiene en los procesos de enseñanza-aprendizaje de la música, complementada con el uso de los dispositivos móviles. Su desarrollo en las aulas es clave para mejorar el aprendizaje cognitivo.

El término de inteligencia emocional, IE de aquí en adelante, fue acuñado por Peter Salovy y John Mayer, psicólogos de la universidad de Yale, aunque ha sido mundialmente difundida por el psicólogo, filósofo y periodista Daniel Goleman.

La IE es la capacidad de sentir, entender, controlar y modificar, tanto estados anímicos propios como ajenos. Asumir la IE como parte de la metodología del proyecto Crea Banda sonora implica algunos cambios muy importantes en el aula.

- Trabajar de forma colaborativa. El aula no tiene como referencia única al docente sino que todos los asistentes participan de forma activa en su propia educación. Desde esta perspectiva, el alumnado emocionalmente desarrollado, entiende que el tiempo que pasa dentro de un espacio educativo es fundamental y por ello lo asume como propio. Como una segunda consecuencia, aparecen los alumnos-tutores, discentes que en diferentes momentos del aula pueden participar en la ayuda al entendimiento del proceso del resto de compañeros.

- Aprender música de forma empírica. Una de las formas de asegurarnos el desarrollo de los procesos emocionales es que el aprendizaje de esta asignatura sea absolutamente procedimental. A hacer música se aprende haciendo música, no hablando de ella. La realidad docente que esto permite, como el desarrollo del yo emocional interior del alumnado, así como la necesidad de crear grupo en el que tocan juntos, lo que mejora el ambiente social del aula y hacen de la música y la IE un binomio indisoluble.

- Señas de identidad propias. En las edades en las que estamos trabajando, pura adolescencia, la importancia del grupo, de la tribu, del clan, es fundamental. Hasta ahora ese aspecto sólo lo han encontrado reforzado fuera del centro educativo, en sus amigos habituales. Es allí donde se les escucha, donde se les entiende, o por lo menos donde son admitidos tal como son y no tal como se les espera que sean. Pero en nuestro proyecto, se encuentran con otro alumnado dentro del aula de música. Gustos musicales compartidos, o no, pero respetados, jerga del aula, constante modulación de las relaciones sociales entre los miembros del grupo. El inmovilismo del aula convencional en que cada uno juega su rol social de la calle, desaparece porque en nuestro proyecto es mucho más importante el rol educativo que cada uno termina asignándose, desapareciendo parte de la realidad que les rodea fuera del 
centro de estudios y siendo arropados por nuevas formas de entender la educación y de entender el mundo que la asignatura de música les está ofreciendo.

- La figura del docente en proceso de transformación. En esta apuesta por una nueva educación también se ven involucrados los docentes. El proceso de aprendizaje emocional no va en una única dirección, sino que afecta a ambos lados. Discentes y docentes ven cómo sus roles en aula modulan y caminan hacia nuevos espacios que facilitan un desarrollo cognitivo común. Una buena gestión de las emociones en el aula permitirá una apertura total del alumnado que, además, al encontrarse con los dispositivos móviles como herramientas naturales de aprendizaje, permitirá creer en el centro educativo, creer en un espacio vital en el que quieren y necesitan estar para conseguir actualizarse y entender el futuro. Pero será el docente quien deberá cambiar primero su rol emocional para poder después extrapolarlo y llevarlo al alumnado.

- La importancia de la comunidad. Este cambio experimentado en el docente dentro del aula también afecta positivamente al centro educativo. La estabilidad emocional y social de todo un colegio o un instituto se ve reconstruida, restaurada y aparecen relaciones educativas totalmente necesarias para el óptimo funcionamiento del mismo. No puede haber educación sin Comunidad Educativa. La gestión de emociones en el claustro permite la ubicación necesaria para los egos, los miedos y los conflictos, facilitando así la creación de redes, de grupos de trabajo muchísimos más amplios que, posteriormente, no tienen por qué ser de un mismo centro, municipio, región o país.

La inteligencia emocional afecta positivamente a la inteligencia social, donde todos participan, todos son protagonistas o líderes en algún momento del proceso y culmina en la inteligencia cognitiva, gracias al aumento del interés por la asignatura de música, la atención en las aulas y el esfuerzo de todos.

\subsection{Inteligencias múltiples}

Comenzamos con palabras del Manifiesto Crea Banda Sonora, elaborado por Antonio Domingo, uno de los directores del proyecto, para entender la importancia que tiene este aspecto en el proyecto.

Para la metodología CBS, acrónimo de nuestro proyecto, hay que educar de forma integral, al ser humano al completo, alimentando cada uno de sus recovecos neuronales. Educar teniendo en cuenta a cada ser humano que habita en nuestro centro educativo, a cada una de sus diferencias, y a cada una de sus habilidades. Educar teniendo en cuenta que TODOS LOS SERES HUMANOS somos un conjunto de Inteligencias Múltiples, (IM a partir de este momento). No sólo existen niños y niñas matemáticos que serán grandes investigadores, o lingüistas que llegarán a ser grandes escritores, o cinéticocorporales que serán colmados con medallas olímpicas. También los hay con habilidades espaciales que serán grandes exploradores, o habilidades 
interpersonales que llegarán a liderar grandes partidos políticos, o habilidades intrapersonales creadores de nuevas líneas filosóficas, o incluso los hay con habilidades musicales, con las que crearán melodías que serán recordadas por los siglos de los siglos. Y todo ello, unido, es un ser humano completo, ese al que en nuestra metodología nos proponemos sensibilizar hacia el camino de la educación. Un ser humano con siete inteligencias de las cuales, ninguna podemos dejar en la cuneta, porque tenemos la misión de desarrollar mentes completas, hombres y mujeres cuyo potencial haya sido explorado al $100 \%$. (Domingo, 2015)

Para ello, para ese desarrollo integral de nuestro alumnado, contamos con la mejor de las herramientas: la música. Dentro de la teoría de Howard Gardner es una de las IM, concretamente la primera que describe en su libro, pero su importancia va más allá del mero hecho de cuál es su lugar expositivo.

Hablaremos de la Inteligencia Musical en el proyecto Crea Banda Sonora como aquella que aglutina a todas y cada una del resto de las IM. Música como lenguaje que desarrolla la capacidad lingüística de nuestro alumnado. Música como construcción matemática, donde los acordes, el ritmo, la armonía, la estructura formal, o el fenómeno físico armónico son elementos numéricos que relacionan música y física. Música como herramienta neuroplástica que desarrolla la psicomotricidad necesaria de la Inteligencia Cinético-Corporal. Música como capacidad para usar el espacio dentro de la composición, para asumir el movimiento del intérprete a la hora de ofrecer sus resultados sonoros en el aula 0 en un concierto. Música como síntesis de lo social y lo emocional, como parte de un grupo que tocan juntos y como aportación propia de creación sonora, acercándonos así a las Inteligencias Interpersonal e Intrapersonal. La música como un punto de apoyo donde la educación al completo gira.

\subsection{Emprendimiento en el aula. Clases orquesta}

Todo ello desde una perspectiva integradora, en el que el alumnado es reconocido como sujeto social, crítico, autocrítico y propositivo, que identifica una situación problemática y es capaz de activar mecanismos de gestión para la resolución de la misma, creando comunidad. El proyecto en sí, permite al alumnado gestionar su propia "empresa" u orquesta, en la que, se siente protagonista dando solución de forma colaborativa y transformando la educación musical tradicional, desde una perspectiva horizontal e integradora. Convertimos para ello todas las clases en una gran orquesta o grupo musical, en el que cada uno asume un rol distinto: cantantes, intérpretes, compositores, letristas, editores de blog o redes sociales, gestores financieros, cartelería, maquillaje...cada uno con capacidades y perfiles distintos que elaboran un producto musical final que exponen delante de sus compañeros del proyecto en un concierto final. Cada uno es importante en cuanto a su participación y colaboración para que la orquesta se desarrolle plenamente. En definitiva estamos desarrollando en el alumnado la inteligencia emocional, todas las inteligencias múltiples, la creatividad, la autogestión de su conocimiento, la confianza en sí 
mismo, la superación del fracaso y el disfrute del éxito, la motivación y el liderazgo, todo ello cooperando y colaborando en equipo.

\subsection{Trabajo por proyectos o colaborativos}

Forma parte de la filosofía del proyecto Crea banda sonora desde el principio y entronca con la inteligencia emocional. Es un proceso de aprendizaje en el que el alumnado asume un rol dentro de la orquesta que le permite motivarse en la búsqueda, producción o creación de nuevos conocimientos a través del trabajo sobre un tema determinado, propuesto por el propio profesor o por el grupo-clase de forma consensuada; en el proyecto que emprende de forma autónoma expone sus necesidades, su criterio propio y, en definitiva sus ilusiones y emociones, todo ello en un proceso de trabajo colaborativo para alcanzar un fin, en su propia educación musical, que culmina en un concierto final. "...a este ejercicio intencional, se le denomina aprendizaje de la autorregulación, que es un proceso autodirigido a través del cual, los aprendices transforman sus capacidades mentales en habilidades académicas." (Maldonado, 2008: 159) De esta manera, todo el alumnado se siente importante dentro de la orquesta y todos tienen una función que complementa a las demás. En definitiva, un proyecto común que se desarrolla con la colaboración de todos. Su base sería el conectivismo, nutriendo y manteniendo conexiones para facilitar el aprendizaje continuo.

\subsection{Educación formal, informal y no formal}

La utilización de dispositivos móviles en el proyecto Crea la banda sonora de tu vida, nos está permitiendo unificar estos tres tipos de educación, que si bien ahora pasamos a diferenciar, se funden en una sola modalidad de educación en la práctica de música en el aula. La educación formal es sistemática, con una intención deliberada y reglada que se concretiza en un currículo oficial y a la que se debe de ajustar todo el profesorado en nuestro país; posee un horario fijo y un calendario definido y se suele ofrecer en un centro de formación que concluye con un título académico. La educación informal es más bien un proceso de aprendizaje espontáneo, no intencional y que se produce en interacción con el mundo que nos rodea. Y la educación formal se obtiene a través de grupos u organizaciones comunitarias ajenas al centro oficial de formación, sin certificaciones ni estructuras. Es difícil en nuestra actual sociedad diferenciar la educación no formal e informal, dándose una continuidad entre las tres en una especie de complementariedad o arquitectura pedagógica.

En el proyecto, es difícil diferenciarlas, puesto que va más allá del aula de música, con continuas interacciones fuera del horario escolar, con actividades realizadas fuera del aula y todo lo que se comparte a través de las redes sociales, sobre todo por el profesorado del proyecto.

\subsection{Mobile Learning}


Básicamente, el Mobile Learning es la utilización de dispositivos o tecnologías móviles como herramienta educativa al servicio de los procesos de enseñanza-aprendizaje. Es una evolución natural de las tecnologías de la información y la comunicación, un dispositivo pequeño que tiene conexión permanente con la red, con altas prestaciones multimedia.

Según Brazuelo F. y Gallego D. (2011, p. 17), podemos definir el Mobile Learning como la modalidad educativa que facilita la construcción del conocimiento, la resolución de problemas de aprendizaje y el desarrollo de destrezas o habilidades diversas de forma autónoma y ubicua gracias a la mediación de dispositivos móviles portables.

"El mobile learning es la intersección de la computación móvil y el eLearning, la cual se caracteriza por la capacidad de acceder a recursos de aprendizaje desde cualquier lugar, en cualquier momento, con altas capacidades de búsqueda, alta interacción, alto soporte para un aprendizaje efectivo y una constante valoración basada en el desempeño".

Se puede entender el Mobile Learning como una extensión del eLearning, pero se diferencia de éste por la ubicuidad y por la alta interactividad y conectividad que poseen los dispositivos móviles.

Para no extendernos, las características tecnológicas asociadas al Mobile Learning son: su portabilidad, ubicuidad, inmediatez y conectividad y la adaptabilidad al usuario, tanto de servicios como aplicaciones e interfaces.

Aunque el aprendizaje móvil es todavía un aprendizaje emergente, es posible encontrar diversas propuestas que ponen en consideración distintos aspectos que se han de tener en cuenta a la hora de diseñar los procesos de enseñanza-aprendizaje.

Estas propuestas se suelen enmarcar dentro de las cuatro categorías que plantea Winters (2006, pp. 5-9):

- Tecno-céntricas: basadas en aprender el manejo de un dispositivo móvil.

- Relacionadas con el E-learning: entendido el aprendizaje móvil como una extensión del E-Learning

- Extensión de la educación formal: se preguntan si en el aprendizaje móvil se pueden desarrollar las mismas estrategias que en la enseñanza tradicional.

- Aprendizajes centrados en los estudiantes: entendiendo el aprendizaje móvil desde la perspectiva del aprendizaje permanente, del protagonismo del alumnado y de su movilidad y la comunicación con el contexto.

Desde el punto de vista pedagógico del Mobile Learning, ofrecemos a continuación ventajas y desventajas que dividiremos en dos tipos: funcional y pedagógico.

- Ventajas:

○ Funcional:

- Aprendizaje en cualquier momento y en cualquier lugar, dando lugar a un aprendizaje más personalizado, permanente, interactivo. Ubicuidad 
- Está al alcance de casi todos, dándose la circunstancia que casi el $100 \%$ del alumnado de ESO, posee un móvil.

- Favorecen el aprendizaje exploratorio, aprendiendo sobre el terreno, experimentando.

- Mayor accesibilidad, conectándose a través de la wifi del centro o con paquete de datos con servicios de Internet.

- Es más barato que un ordenador portátil o un PC

- Mayor portabilidad y funcionalidad.

- Destreza en el manejo por parte del alumnado

- Enriquecimiento multimedia del aprendizaje

- Pedagógico:

- Mejora las competencias lectoras y matemáticas

- Motiva a realizar experiencias de aprendizaje tanto individuales como grupales

- Favorece el aprendizaje colaborativo, exploratorio y activo

- Ayuda a identificar carencias de aprendizaje en el alumnado

- Mejora la competencia tecnológica e informacional

- Mantiene un alto nivel de interés y motivación en el alumnado

- Promociona el aprendizaje flexible

- Mejora la interacción didáctica de forma síncrona y asíncrona

- Diversidad: ante alumnado heterogéneo hay que dar soluciones heterogéneas y los dispositivos móviles proporcionan personalización e individualización en el aprendizaje.

- Favorece el aprendizaje la pantalla táctil de los dispositivos móviles al alumnado con necesidades educativas especiales, ya que posee aplicaciones específicas y opciones de accesibilidad.

- Ofrece respuestas y evaluaciones inmediatas

- Aprendizaje contextual y realidad aumentada

- Mayor alcance e igualdad de oportunidades

- Vínculo entre la educación formal, informal y no formal

- Habilita nuevos entornos de aprendizaje

- Desventajas:

○ Funcional:

- Pantallas pequeñas

- Dificultad en generar sistemas complejos

- Falta de autonomía, la batería de los dispositivos móviles, con un uso intensivo, suelen durar muy poco y si hay que cargarlos perdemos el concepto de móvil.

- Escaso espacio de almacenamiento e incompatibilidad entre sistemas, por ejemplo iOS y Android.

- Costoso procesamiento computacional

- Teclados pequeños

- Precio. La tecnología cambia continuamente y los dispositivos móviles habría que actualizarlos con frecuencia (obsolescencia programada)

- Pérdida o robo de los dispositivos móviles 
Alises Camacho, Ma Encarnación. Potencial pedagógico del Mobile Learning en el aula de música en secundaria

- Pedagógico:

- Si no son bien utilizados, pueden suponer una distracción en el aprendizaje

- Accesibilidad. Si el profesorado no está familiarizado con los estándares de accesibilidad, pueden perjudicar o aislar al alumnado, sobre todo a los que tienen problemas motrices.

- Falta de criterios uniformes para la enseñanza-aprendizaje con dispositivos móviles.

- Adaptar pedagogías tradicionales a la realización de actividades con dispositivos móviles.

- Posibles dificultades en su implantación debido a resistencias de las familias y/o de las instituciones públicas.

Como se puede apreciar, son más las ventajas que nos ofrece la utilización de dispositivos móviles en la enseñanza, que desventajas, que son más de tipo funcional y que, con el avance y desarrollo de estos, se pueden solucionar. El Mobile Learning está presente en el proyecto Crea la banda sonora de tu vida, para paliar, en parte, la falta de recursos musicales en las aulas de los centros de Castilla La Mancha y para aprovechar el gran potencial creativo que poseen y su posibilidad de compartir contenido de forma inmediata a través de redes sociales.

\subsection{Aspecto relacional de los dispositivos móviles}

Los dispositivos móviles tienen una base tecnológica relacional evidente. En los medios comunicacionales transmedia actuales, la presencia de los dispositivos móviles supone una gran plataforma de interacción muy potente, sobre todo, desde la redes sociales, donde se da un consumo ubicuo tanto de productos narrativos como musicales, donde se comparte contenido y se produce un remixado constante, desde un consumo multipantalla. El alumnado de Secundaria utiliza el dispositivo móvil, sobre todo, para comunicarse, para compartir contenidos audiovisuales y se ha convertido en una herramienta indispensable en su quehacer diario.

...estamos inmersos en la creación de un modelo social y democrático hacker que pretende hacer primar la persona y la acción colaborativa, con la implementación de un liderazgo que trasciende lo individual para convertirse en compartido y colectivo, aprovechando la potencialidad de las redes sociales. (Marfil-Carmona et al., 2015: 34).

El proyecto genera interacciones y comunicación constantes, desde un remixado continuo, entre sus integrantes, tanto profesorado como alumnado, con la utilización de los dispositivos móviles en el aula de música. Pasamos por tanto, de hablar de TIC, tecnologías de la información y de la comunicación a TRIC, añadimos la R de relación, en el que "el concepto de TRIC supera el mero determinismo tecnológico, el término relación imbrica todo el potencial de la multialfabetización que se produce en 
Alises Camacho, Ma Encarnación. Potencial pedagógico del Mobile Learning en el aula de música en secundaria

las interacciones en el plano creativo y en la dimensión receptiva de cada uno de los coautores o mediadores." (Gabelas et al., 2012)

Pierre Lévy considera el ciberespacio un «intelecto colectivo» o suma de inteligencias compartidas que crean una especie de cerebro común. Este autor afirma que los entornos virtuales son el espacio conversacional por excelencia, donde las acciones colaborativas van a llevar a los individuos a la construcción de su conocimiento. Por su parte, Howard Rheingold propone el término Multitudes Inteligentes para definir la organización social de las personas que participan en el ciberespacio donde, sin una organización explícita, coexisten y actúan colectivamente. Lo más importante es que la interactividad de los individuos en el espacio digital va a crear un saber colectivo sin que, en muchos casos, exista una intencionalidad expresa de crear dicho saber colectivo. (Osuna et al., 2012, p. 7)

\section{OBJETIVOS}

A través del presente estudio se ha tratado de:

- Conocer y valorar las implicaciones educativas, pedagógicas y comunicativas de los entornos digitales móviles en el aula de música de Secundaria en centros públicos de Castilla La Mancha del proyecto Crea la banda sonora.

- Identificar las competencias que se desarrollan con el uso de móviles y tablets en el aula de música.

- Demostrar la eficacia de un sistema de formación basado en entornos digitales.

- Conocer la experiencia, vivencia y/o sugerencias de alumnado y profesorado sobre el uso y producción de música con dispositivos móviles

- Proponer un modelo de enseñanza-aprendizaje, un paradigma educativo o una teoría de aprendizaje, que teniendo como base los entornos digitales móviles, emerja de la reflexión sobre la práctica docente del profesorado estudiado y del aprendizaje del alumnado.

\section{METODOLOGÍA}

Elegimos para nuestro estudio una técnica cualitativa de triangulación, llamada de Investigación-Acción participativa o colaborativa, que se complementó con encuestas de corte cuantitativo y con una visión crítica de la práctica educativa con dispositivos móviles, a través de entrevistas a personas expertas en el tema; con foros de discusión tanto con el profesorado voluntario que participó también en las encuestas, como con el equipo pedagógico del proyecto y fichas de seguimiento a pie de aula.

Esta metodología de Investigación-Acción se ajustaba a los objetivos planteados desde el principio del estudio, puesto que era una forma de estudiar y explorar una situación educativa concreta, con la finalidad de mejorarla y en la que se implican como indagadores, los participantes en la realidad investigada. Todo ello nos permitió identificar diversas estrategias de acción que se fueron implementando durante la investigación y que eran sometidas continuamente a observación, 
reflexión y cambio. Se dividió en dos cursos escolares: 2014-2015 y 2015-2016 y en cada uno de ellos se desarrollaron seis fases en continua retroalimentación: diagnóstico, planteamiento del problema y objetivos, plan de acción, evaluación, resultados e implicaciones y propuesta de acción.

Además, desde el Centro de Formación del Profesorado, se proporcionó formación tanto técnica como metodológica, al profesorado implicado en el estudio, por profesionales competentes en estas materias. (Aprendizaje Basado en Proyectos, Inteligencias Múltiples y Emocional, Aplicaciones musicales y educativas en dispositivos móviles, claves para incorporar el Mobile Learning en educación, el reto de formar smartusers, relación entre redes sociales, dispositivos móviles y pedagogías emergentes)

Desglosamos brevemente los instrumentos utilizados y su muestra:

- En cuanto a las encuestas, diseñada con preguntas cerradas y Escalas de Likert, se realizaron las siguientes:

- Alumnado: dos, al inicio y final del curso 2014-2015, con 350 y 100 participantes respectivamente, sobre competencia informacional y uso de entornos móviles y en concreto, en la asignatura de música y dentro del proyecto Crea Banda Sonora.

- Profesorado, igualmente dos al inicio y final del curso 2014-2015, con 11 y 9 participantes respectivamente, sobre el mismo tema indicado con anterioridad, uso de las TRIC en la asignatura de música y sobre el desarrollo de competencias e implicaciones educativas, pedagógicas y comunicativas con entornos móviles, incluyendo la valoración y puesta en práctica de los seis pilares pedagógicos del proyecto.

- Encuesta al profesorado, sobre el modelo TPACK y su desarrollo en el aula como marco para diseñar experiencias de enseñanza-aprendizaje con 28 participantes y otra encuesta sobre el marco de Coomey y Stephenson con otros 20 participantes, durante el curso 2015-2016. Estas encuestas se realizaron después del segundo foro de discusión, en el que todo el equipo pedagógico decidió realizar una encuestas sobres estos dos marcos que se ajustaban mejor al proyecto.

- El análisis cualitativo se realizó con los siguientes instrumentos:

- Durante el curso 2014-2015, en el tercer trimestre, el profesorado realizó fichas de seguimiento en el aula, con 42 fichas realizadas y 7 centros participantes, con una serie de ítems que permitía valorar desde la organización de la sesión, actividad del docente y del alumnado y la integración de los dispositivos móviles en los procesos de enseñanza-aprendizaje. Además de contar con material audiovisual de las producciones finales.

- Se realizaron durante los dos cursos escolares, 5 entrevistas a expertos en la materia, (Tíscar Lara, Adolf Murillo, Pilar Soro, José Luis Miralles y 
Andrea Giráldez), que versaba sobre el concepto de Mobile Learning del que hemos entresacado una definición, la situación actual en nuestro sistema educativo, estrategias, ventajas y desventajas de su implantación en los centros escolares de Secundaria, destrezas y competencias necesarias en profesorado y alumnado para que sea una herramienta educativa válida y a nivel pedagógico, qué paradigma o modelo de enseñanza-aprendizaje se ajustaba más al Mobile Learning, valorando los seis pilares pedagógicos del proyecto.

- Dos foros de discusión sobre modelos, metodologías y marcos de diseño de experiencias de enseñanza-aprendizaje con el equipo pedagógico del proyecto, en total 8 personas, durante el curso 20152016, que como hemos comentado anteriormente, hicieron dos propuestas de encuestas para profesorado.

- Los resultados que recogimos durante el curso 2014-2015, fueron presentados en el encuentro que tuvo lugar en Cuenca, en Octubre de 2015, a toda la comunidad Crea Banda Sonora y nos sirvió como punto de partida para comenzar a trabajar con el equipo pedagógico del proyecto, de cara al curso 2015-2016.

- El equipo pedagógico elaboró de forma consensuada una Taxonomía de Bloom con las apps educativas y musicales que el profesorado del proyecto utiliza en su aula de música con frecuencia.

\section{ANÁLISIS Y DISCUSIONES}

Los dispositivos móviles se han convertido en una herramienta fundamental, indispensable para la mayoría de la sociedad actual. Hemos recogido datos de numerosos proyectos en la parte teórica del estudio, que nos permiten afirmar que para los adolescentes es como una extensión de su mano, y son herramientas que controlan y dominan técnicamente, mejor que sus propios profesores. Además, es una herramienta que les motiva a aprender, les permite comunicarse, refuerza la innovación y el pensamiento crítico desde un aprendizaje ubicuo y flexible que contrastan con los datos de nuestro estudio.

Una herramienta muy potente, con una tecnología muy avanzada y en continuo progreso, tanto en tecnología de acceso como de localización, con unas evoluciones técnicas de aplicaciones como de servicios móviles o de sistemas operativos, que nos han obligado a revisar y actualizar constantemente la parte teórica. En un futuro inmediato, se mejorará el rendimiento de los dispositivos móviles en cuanto a hardware, con móviles ultrafinos, que funcionarán con gestos y comandos de voz y se utilizarán como equipo de telecomunicaciones, como un ordenador multimedia e incluso como mando a distancia, que favorece lo que se llama la Internet de las cosas, donde todo estará interconectado.

La tecnología ha estado presente en la Música, desde sus orígenes, con la evolución constante de los instrumentos musicales o la invención de los sistemas de reproducción y grabación de sonido, que han supuesto una auténtica revolución en los sistemas de producción, interpretación, composición y consumo musical. Y para la 
asignatura de música, el estudio demuestra la cantidad de apps que se pueden utilizar en el aula, tanto para crear entornos personales de aprendizaje, como su uso como instrumento musical, la interpretación o la creación, no sólo de música, sino de material audiovisual, en la que mezclar música, arte y tecnología, junto al resto de las asignaturas del currículo oficial en Castilla La Mancha. El desarrollo tecnológico ha ido modificando los referentes musicales: escucha simultánea, reconocimiento al instante de música o la forma de compartirla masivamente en la red. No aprovechar los recursos y herramientas tecnológicos que existen para la asignatura de música, supondría no dar una respuesta adecuada a las demandas sociales de nuestra sociedad de la información y la comunicación. Una asignatura de música que es un elemento esencial en la formación de cualquier individuo, desarrollada por grandes pedagogos y estudiada actualmente por la neurociencia con resultados sorprendentes, puesto que es la materia que más zonas activa en el cerebro humano, sobre todo, a nivel emocional.

A pesar de estas consideraciones, hay voces que se levantan contra su uso a nivel educativo e incluso los prohíben en sus centros. Las razones que esgrimen es que son fuentes de distracción en el aula y promueven la desigualdad económica del alumnado. Este segundo aspecto se cae por sí solo al demostrar que en el alumnado del proyecto encuestado y en otros estudios presentados, en un $97 \%$ poseen dispositivos móviles, como veremos más abajo en la figura 2.

Con respecto al tema de la distracción y la poca atención, hemos de señalar que para que los dispositivos móviles sean una herramienta y un recurso pedagógico útil en el aula, el profesor debe de jugar un papel fundamental como mediador y guía en los procesos de enseñanza-aprendizaje, propiciando un cambio metodológico en el aula, más allá de su uso, creando experiencias significativas y facilitando recursos e información en tiempo real al alumnado. Como veremos más abajo, en el proyecto CBS se trabaja en este sentido, pero no en todos los proyectos escolares se hace este planteamiento pedagógico. El problema no es la tecnología, sino la metodología y cómo trabajamos los contenidos en el aula. Es cierto que nuestro alumnado pasa muchas más horas delante de una pantalla que en la escuela; la diferencia estriba en que lo empleen educativamente o sólo como entretenimiento y ahí es donde el profesorado tiene que enseñar a hacerlo adecuadamente.

Los ejecutivos de las compañías más potentes a nivel tecnológico de Silicon Valley, envían a sus hijos e hijas a la escuela Waldorf School of the Peninsula, una escuela donde no hay pantallas de ningún tipo. La razón que esgrimen es que van en detrimento de su capacidad para crear una conexión significativa con los demás y el mundo que les rodea. Es otro reto para la escuela, enseñar al alumnado a crear conexiones significativas en tiempo real y virtual. Se relaciona constructivismo con Mobile Learning (Scopeo, 2011), propiciando una interacción social que permite construir conocimiento socialmente, permitiendo al alumnado construir un andamiaje desde el aprendizaje significativo. Señala además, que el Mobile Learning plantea la necesidad de generar espacios de construcción e intercambio de información.

En un informe sobre las tendencias que impulsarán cambios en la educación universitaria (NMC Horizon Report, 2014), nos señalan la integración del aprendizaje 
colaborativo, en línea e híbrido; la ubicuidad en medios sociales; prosumidores de contenido, aprendizaje y evaluación a través de datos, enfoques flexibles. ¿Cómo llegará nuestro alumnado a estos cambios, si no los hemos preparado antes en la escuela primaria y secundaria? ¿Cómo accederán a nuevos puestos de trabajo que existirán en unos años, si no les hemos educado en el uso de las tecnologías tan cambiantes y en constante evolución?

En la situación actual del Mobile Learning en España y en la etapa educativa de ESO, parece que se ha dado un paso importante pero no suficiente para su implantación. Hay proyectos, pero aún muy aislados, sin un respaldo ni de instituciones, ni de los centros, que están muy por detrás de la tecnología que utiliza nuestro alumnado. Hace falta, por tanto un diseño integral, diseños más fundamentados, con estructuras claras, facilitando su incorporación en las aulas con ayuda, formación, permitiendo una penetración más global. Además, hay que adoptar metodologías adecuadas, una pedagogía clara y compartida, para que los dispositivos móviles mejoren los procesos y enfoques docentes; para hacer lo mismo que hasta ahora, no tiene sentido incorporarlos al aula.

Un aspecto que se ha señalado continuamente en nuestro estudio como importante ha sido el de la motivación. Para el alumnado, la utilización de dispositivos móviles y el proyecto Crea banda sonora, es muy positivo y ha supuesto una visión de la clase de música distinta, que les motiva más a aprender. El concierto final que se realiza y los conciertos de centro, son una motivación extra y lo señalan como uno de los aspectos más importantes del proyecto, junto al reparto de roles. Señalar también, que un $44,3 \%$ está de acuerdo en que mejora el clima de clase y que un $34,9 \%$ señala que le ha ayudado a mejorar sus notas en algunas asignaturas, un $9,1 \%$ en todas. Lo refrendan estudios que hemos recogido: la gran mayoría de los estudiantes creen que los dispositivos móviles tendrán un impacto positivo en el aprendizaje, resulta más divertido y aligera sus mochilas. (Pearson Student Mobile Device, 2013).

Insistimos en que con un uso pedagógico y metodológico adecuado, junto a la motivación, puede hacer de los dispositivos móviles una herramienta muy potente en educación. Hemos de considerar la diferencia de uso que manifiestan en las encuestas, entre profesorado y alumnado. El alumnado tiene más intuición en el uso y manejo de los dispositivos móviles que el profesorado, (hay cierto miedo en el profesorado en el dominio de esta tecnología), pero el profesorado ha manifestado un dominio de su materia y de las metodologías suficiente para acometer una enseñanza adecuada con los dispositivos móviles, mostrando una mente abierta a que el mismo alumnado le enseñe ese uso más intuitivo. El profesorado debe de ser en todo momento facilitador en los procesos de enseñanza-aprendizaje, quedando demostrado que prima en ellos el aprendizaje significativo, activo.

El profesorado CBS, tienen un porcentaje muy alto en cuanto conocimiento tecnológico, con un $65 \%$ que dice tener un conocimiento real de los dispositivos móviles y que saben resolver problemas técnicos. Un $70 \%$ afirma que sabe seleccionar nuevos enfoques docentes y metodologías activas para guiar el aprendizaje del alumnado. 
Una adecuada formación tanto tecnológica, como pedagógica y metodológica es urgente en el profesorado de nuestras escuelas. Hemos señalado durante nuestro estudio que es importante tener en consideración el modelo TPACK y su aplicación en las aulas. El profesorado actual, en general, no tiene una formación continua, sobre todo en metodologías activas y en uso de las TRIC, con lo cual se hace difícil una adopción de los dispositivos móviles en el aula.

Un $80 \%$ del alumnado afirma que la utilización de los dispositivos móviles favorece el aprendizaje, aunque sólo un $52 \%$ está de acuerdo en usarlo para ello. Si nos centramos en el aula de música, su uso favorece los aspectos de componer e interpretar música, buscar y compartir información, gestionar tareas y el trabajo colaborativo. Es la asignatura en la que más usan el dispositivo móvil, dato evidente porque participan en el proyecto, pero llama la atención que en las asignaturas troncales, prácticamente no se utilicen.

Se hace indispensable, según estos datos, una adopción a nivel de todo el claustro de profesorado en las escuelas, de estas metodologías activas, que tengan una fundamentación pedagógica y reflexiva sobre los procesos de enseñanza aprendizaje, con un compromiso a nivel institucional, tanto nacional como regional de facilitar esta formación, no solo la aportación de material tecnológico, como ha sucedido en los últimos años, por ejemplo, con el proyecto Althia en Castilla La Mancha. El profesorado Crea Banda Sonora, se ve aislado en la puesta en práctica del proyecto, porque el resto sigue enseñando desde una visión más tradicional de la enseñanza.

Otro aspecto que nos lleva a discusión, es el de la integración de educación formal, informal y no formal. En nuestras escuelas se da demasiada importancia a la formal, sin que se esté produciendo una integración con las otras dos. Hemos señalado en la parte teórica que es necesaria la integración de las tres, debido al cambio vertiginoso y no siempre previsible de la realidad social actual, porque la vida humana es un ciclo de permanente formación y construcción de la persona en la que el individuo está en constante aprendizaje y porque la realidad de los proyectos estudiados y nuestro propio estudio, nos muestran a un alumnado en constante aprendizaje más allá de las aulas, a las que asisten muy pocas horas, y en la que están utilizando los dispositivos móviles sin una orientación pedagógica encaminada al aprendizaje.

Para finalizar este apartado de discusiones, señalamos que no hemos encontrado ningún estudio o proyecto con dispositivos móviles, que trate el aspecto crítico de la utilización de estos en el aula, de forma objetiva. Nuestro estudio ha demostrado que el aspecto crítico no se trabaja suficientemente en el aula de música o, al menos, de forma explícita, a pesar de que se han modificado los planes de estudio hacia el desarrollo de competencias, sobre todo, la de aprender a aprender, porque la enseñanza actual se sigue apoyando en un enfoque orientado principalmente a la adquisición de contenidos. El equipo pedagógico del proyecto ha pretendido con la taxonomía de Bloom que realizó de forma consensuada con las apps móviles, que el alumnado llegue a categorías de pensamiento superior, pero en la práctica se ha reflejado que no se es consciente 0 no se planifica suficientemente sobre este supuesto. Este pensamiento de orden superior supone una fusión tanto del pensamiento crítico como creativo y se refuerzan mutuamente y es imprescindible en 
la enseñanza del s. XXI. Hemos revisado las pedagogías emergentes actuales y las hemos contrastado con nuestro proyecto y este aspecto nos ayudará a aplicar el pensamiento crítico en él.

Vamos a desglosar de forma más detallada los aspectos que venimos comentando hasta ahora en este apartado.

Las encuestas nos permitieron conocer datos objetivos en cuanto a edad, curso y sexo del alumnado participante: mayoritariamente de $2^{\circ}$ y $3^{\circ} \mathrm{ESO}, 56 \%$ de género femenino y un $44 \%$, masculino. En cuanto al profesorado, la media de edad comprendía entre 36-45 años y un $91 \%$ poseía licenciatura, con más de 5 años de servicio activo, es decir, que tiene ya experiencia en la enseñanza y está preparado para impartir su asignatura.

Figura 1

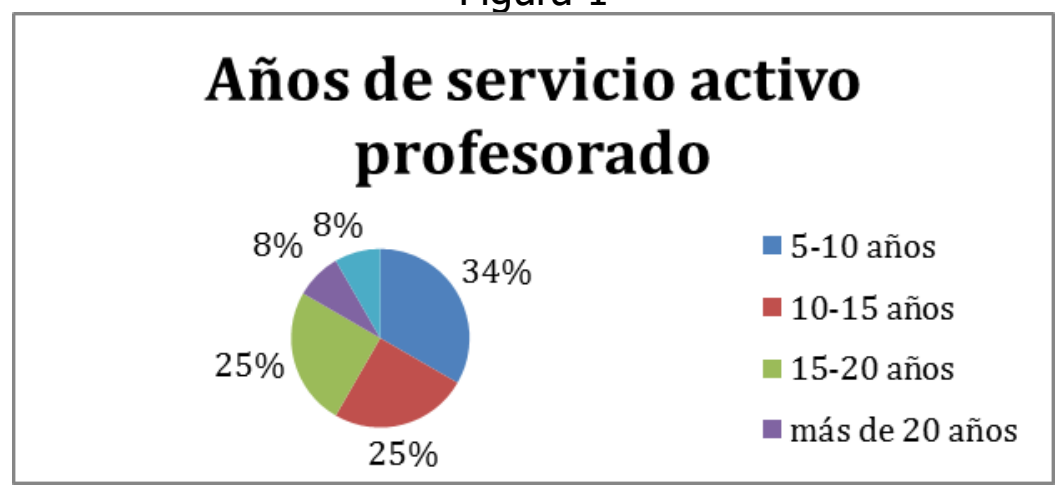

Fuente: Elaboración propia

Partimos en el estudio de unos datos contundentes en cuanto a utilización del dispositivo móvil por parte del alumnado del proyecto en el aula de música, con un $97 \%$ y del profesorado con un $91 \%$ móviles y un $87 \%$ tablets y en el que el sistema operativo más utilizado es Android. Un $69 \%$ del alumnado tiene conexión a internet de forma permanente, aunque hemos de decir que casi todas las apps musicales, no necesitan conexión a Internet para funcionar, sólo hay que descargarlas previamente, excepto las que son redes sociales, en las que se comparte la creación o remixado de piezas musicales. En general, el alumnado lleva su dispositivo móvil al aula de música, en clases BYOD, trae tu propio dispositivo, puesto que el profesorado posee pocos para utilizar en el aula y normalmente, suelen ser propios. Por tanto, no es excusa la carencia de dispositivos móviles para utilizarlos en el aula, puesto que en estas edades, prácticamente todo el alumnado lo posee. 
Figura 2

\section{Teléfono propio alumnado}

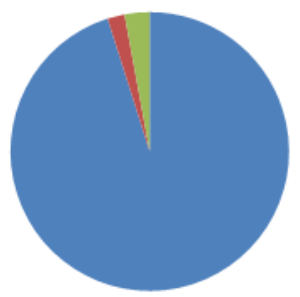

$$
\begin{aligned}
& \text { SÍ } \\
& \text { No } \\
& \text { Utilizo el de un } \\
& \text { familiar o amigo }
\end{aligned}
$$

Fuente. Elaboración propia

Sobre el conocimiento o valoración de las implicaciones educativas, pedagógicas y comunicativas de los dispositivos móviles, el profesorado prima el aprendizaje significativo, activo, basado en el constructivismo y conectivismo, erigiéndose como guía en el aula, en la que el alumnado es el protagonista, comprendiendo su propia personalidad, desarrollando sus potencialidades, desde la creatividad, las inteligencias múltiples, con una continua retroalimentación discente-docente y facilitando la construcción de sus propias redes de aprendizaje, generando pensamiento reflexivo y crítico, sin olvidar la importancia de la inteligencia emocional, las habilidades sociales, la motivación y la empatía. Para el alumnado los dispositivos móviles les han ayudado a desarrollar, sobre todo, las habilidades sociales (recordemos el aspecto relacional que contiene la utilización de los dispositivos móviles), la motivación, la autoconciencia y el control de emociones, además del aprendizaje con dispositivos móviles, con un descubrimiento positivo de las apps musicales y su aspecto práctico y motivador, que les permite realizar creaciones e interpretaciones más motivadoras y ricas.

Figura 3.

Importancia que se da a los seis pilares pedagógicos. Profesorado

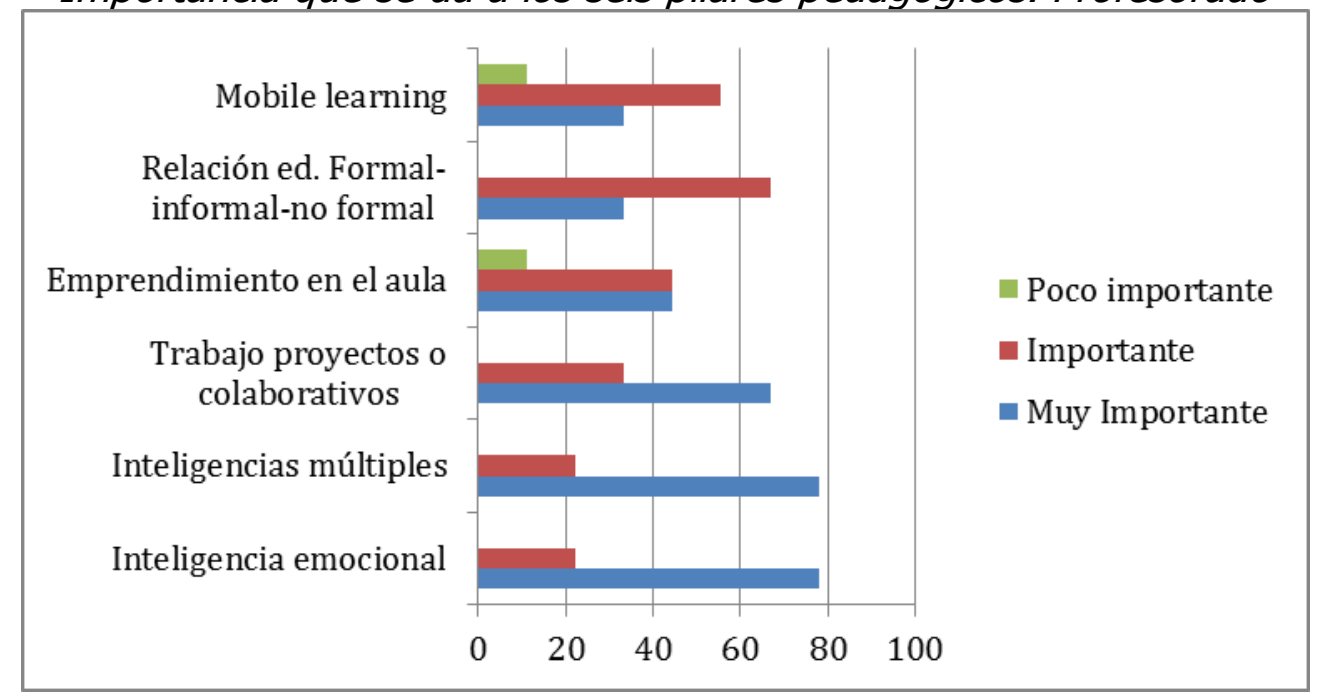

Fuente: elaboración propia 
Figura 4

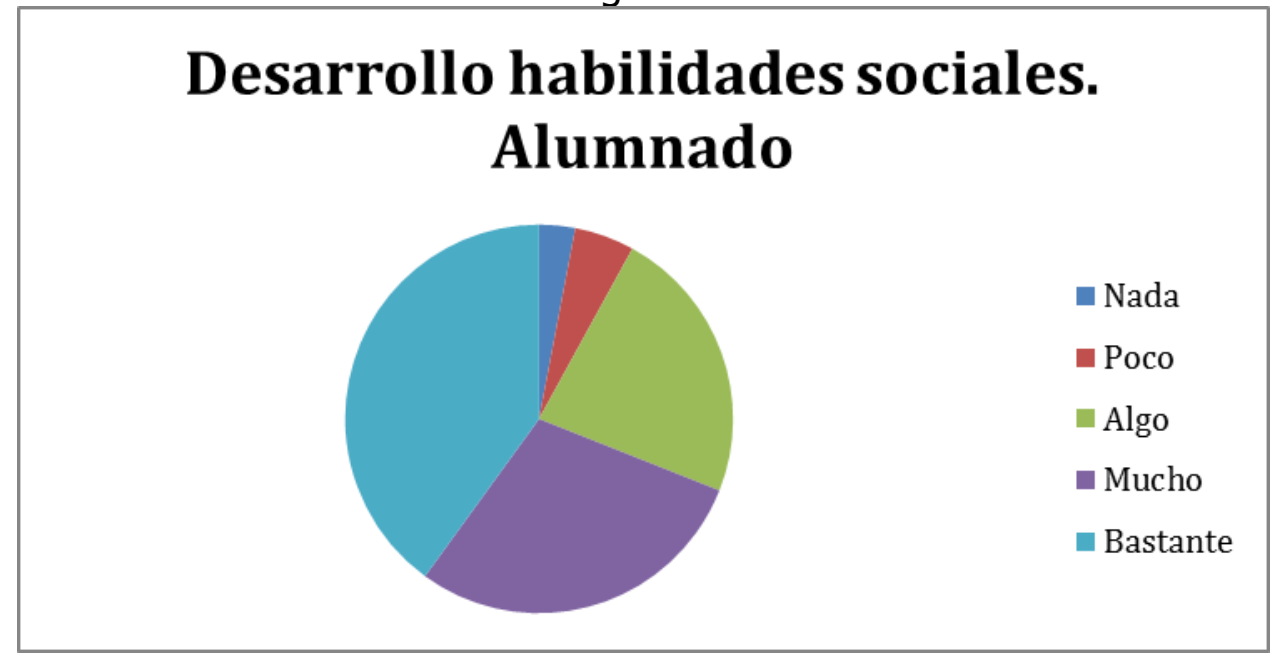

Fuente: Elaboración propia

Los dispositivos móviles, evidentemente, desarrollan la competencia digital, tanto en el conocimiento, selección y configuración de instrumentos y aplicaciones, como en el tratamiento de la información, organización de entornos de trabajo y aprendizaje y la comunicación interpersonal y colaboración, participando en entornos de comunicación interpersonal y publicación en red para compartir información, generando nuevos productos de los ya existentes (mashup). También desarrollan la competencia musical, tanto en la puesta en práctica de contenidos, creando comunidades de aprendizaje (orquestas en el aula), potenciando la improvisación composición e interpretación grupal. El profesorado integra adecuadamente contenido, pedagogía y tecnología (modelo TPACK), conoce y maneja adecuadamente los dispositivos móviles, domina con suficiencia la materia que imparte, en este caso música y tiene una buena base pedagógica en su práctica educativa.

Figura 5

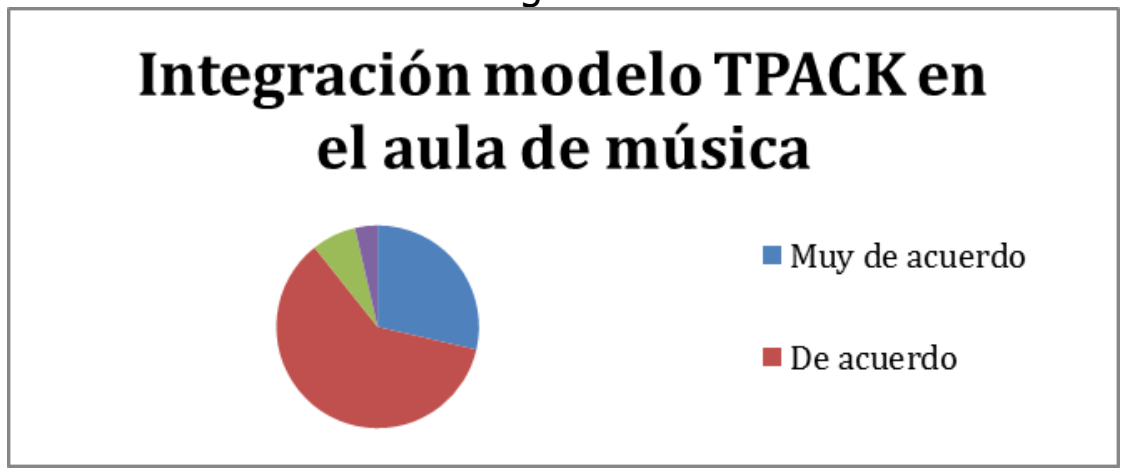

Fuente: Elaboración propia

Insistimos que para el profesorado el Mobile Learning supone una ayuda para comprender aspectos musicales de forma práctica, favorece el trabajo colaborativo y la interpretación colectiva; al haber un reparto de roles en la orquesta, supone una 
mayor implicación emocional, sobre todo en el uso de redes sociales y mejora el comportamiento con una mayor satisfacción y responsabilidad grupal. Las mayores dificultades de su utilización tienen que ver con aspectos técnicos como latencia, conectividad, dificultad para coordinar instrumentos digitales y tradicionales y la gestión eficiente de la información. El alumnado señala que es una gran ayuda para entender los distintos aspectos musicales, sobre todo ayuda en la interpretación y composición musical y para navegar por internet y relacionarse a través de redes sociales.

Resumiendo este apartado, perfilamos el modelo pedagógico en el que se asienta la utilización de los dispositivos móviles sobre el constructivismo y conectivismo, el desarrollo de la inteligencia emocional y las inteligencias múltiples y colectivas, el trabajo por proyectos y colaborativos y el emprendimiento en el aula; aprendizaje distribuido, social, en red y conectado, aprendizaje personalizado, teniendo el modelo TPACK, como el marco más apropiado para diseñar e integrar los objetivos que se persigue con la utilización de los dispositivos móviles en el aula de música en este proyecto. Todo ello consensuado en los foros de discusión por el equipo pedagógico del proyecto y por los expertos entrevistados.

\section{CONCLUSIONES}

Asistimos a una mediamorfosis de los medios, con una reconfiguración de sus usos, con nuevos lenguajes, con unas audiencias que no sólo consumen, sino que están generando conocimiento desde el entretenimiento con diferentes formatos. El Mobile Learning favorece la adquisición de competencias digitales básicas para los profesionales del s. XXI como son el trabajo colaborativo, la comunicación en red, la utilización de fuentes de información diversas y el aprendizaje continuo y ubicuo. Pero la escuela del s. XXI aún está a años luz de trabajar sobre estos pilares en sus aulas. "Se estima que la alfabetización audiovisual y multimedia del profesorado es una asignatura pendiente, previa a la propia alfabetización de los jóvenes, que necesita con carácter de urgencia un tiempo, un espacio y un contenido curricular". Marta et al., 2014, p. 65.

Las investigaciones sobre los dispositivos móviles que contemplamos en nuestro estudio, en su parte teórica, versan normalmente, sobre su difusión y penetración en la sociedad, tanto a nivel mundial como en España, y su valor en el mercado y en el sector de las telecomunicaciones. Otras centran su interés en considerar a los dispositivos móviles como variable dependiente e independiente para poder medir y evaluar los procesos de adopción o su uso en determinados contextos. Por el contrario, son pocos los estudios que se plantean las implicaciones pedagógicas, sociales e interculturales en el ámbito educativo.

Con este estudio hemos intentado demostrar el gran potencial pedagógico de los dispositivos móviles en educación y en concreto, en el aula de música en Secundaria. Nos atrevemos a definir, según todos los resultados obtenidos el Mobile Learning: 
Metodología de enseñanza-aprendizaje o enfoque didáctico que integra los dispositivos móviles como una herramienta de trabajo y en el que se sintetizan contenido-pedagogía y tecnología, ofreciendo un aprendizaje personalizado en cualquier momento y lugar, un aprendizaje conectado, colaborativo y que hace uso de contenidos digitales en abierto; mucho más social, multimedia, desde una cultura en red y que elimina los límites entre el aprendizaje formal e informal.

Se tiene que dar una co-evolución dialéctica entre procesos de aprendizaje y dispositivos móviles para que cumplan su función pedagógica

En los procesos de enseñanza-aprendizaje señalamos como puntos fuertes del proyecto, la co-producción del conocimiento, la interactividad y autonomía, la necesaria relación entre aprendizaje formal-informal y no formal. Es siempre este aprendizaje colectivo, con dinámicas de coaprendizaje e interaprendizaje, construyendo conjuntamente. Se da un adecuado nivel de motivación, por tanto, que favorece el aprendizaje conjunto por experimentación grupal.

Se potencia el aprendizaje contextual y ubicuo, en cualquier momento y lugar, traspasando los límites del aula, convirtiéndose en una educación expandida. Se prima la iniciativa del alumnado, su capacidad creativa y el trabajo colaborativo, favoreciendo la creación de música, a partir del conocimiento que ya existe, mezclando y remezclando hasta llegar a un producto final (mashup). Y se tienen como ejes transversales los seis pilares pedagógicos que hemos ido señalando anteriormente.

Se hace necesario, contrastar este estudio con otros de las mismas características, pero todavía son pocos los existentes, como hemos señalado. Se debe seguir haciendo una reflexión pedagógica más amplia del proyecto Crea Banda sonora, en aspectos como la educación mediática, la inteligencia emocional, el conectivismo, la inclusión educativa, trabajando con mayor profundidad el aspecto crítico del uso de los dispositivos móviles y los avances de la neurociencia, enriqueciéndose con proyectos que ya se están desarrollando en nuestro país como es Soundcool, que integra plástica, música y tecnología con dispositivos móviles, con una formación continua que sea respaldada y auspiciada tanto por las administraciones educativas como por toda la comunidad educativa.

\section{REFERENCIAS}

Brazuelo, F. \& Gallego, D. (2011) Mobile Learning. Los dispositivos móviles como recurso educativo. Sevilla: Editorial MAD, S.L.

Ditrendia. (2016). Informe Mobile en España y en el mundo 2016. Recuperado de http://www.amic.media/media/files/file 352 1050.pdf.

Domingo, A. (2015). Manifiesto CBS. Recuperado de http://goo.gl/mH1zog

Gabelas, J.A.; Marta, C. \& Aranda, Daniel. (2012) Por qué las TRIC y no las TIC. Revista Comein, $9 . \quad$ Recuperado de 
Alises Camacho, Ma Encarnación. Potencial pedagógico del Mobile Learning en el aula de música en secundaria

http://www.uoc.edu/divulgacio/comein/es/numero09/articles/Article-DaniAranda.html

Herrington, J. (2009) Using mobile technologies to develop new ways of teaching and learning. Australia: University of Wollongong.

Isea. (2009). Mobile learning: análisis prospectivo de las potencialidades asociadas al ML. Recuperado de http://goo.gl/vJxrEN

Maldonado Pérez, M. (2008). Aprendizaje basado en proyectos colaborativos. Una experiencia en educación superior. Laurus, 14 (28), 158-180.

Marfil-Carmona, R.; Hergueta, E. \& Villalonga, C. (2015). El factor relacional como elemento estratégico en la comunicación publicitaria. Anàlisi. Quaderns de Comunicació i Cultura, 52, Recuperado de http://www.analisi.cat/index.php/analisi/article/view/n52-marfil-herguetavillalonga/0

Marta, C. \& Gabelas, J.A. (2013). Hábitos de consumo televisivo de ficción entre los universitarios que estudian comunicación. Revista de Comunicación de la SEECI. XVII(31), 14-33. doi:doi.org/10.15198/seeci.2013.31.14-33

Marta, C; Grandío, M \& Gabelas, J.A. (2014). La educación mediática en las titulaciones de Educación y Comunicación de las universidades españolas. Análisis de los recursos recomendados en las guías docentes. Vivat Academia. Revista de Comunicación, 126, 63-78. doi:doi.org/10.15178/va.2014.126.63-78

Monográfico Scopeo No 3. (2011). M-Learning en España, Portugal y América Latina. Recuperado de content/uploads/2013/04/scopeom003.pdf http://scopeo.usal.es/wp-

NMC Horizon Report 2014 Higuer Education Edition. (2014). Recuperado de http://www.nmc.org/publications/2014-horizon-report-higher-ed

Osuna, S; Mata, C \& Aparici, R. (2012) Valores de la formación universitaria de los comunicadores en la Sociedad Digital: más allá del aprendizaje tecnológico, hacia un modelo comunicativo. Razón y Palabra, 17(81). Recuperado de http://www.redalyc.org/pdf/1995/199524700032.pdf.

Pearson. Student Mobile Device (2013). Students in grades 4-12. Recuperado de http://goo.gl/t4k3J7

Winters, N. (2006). What is mobile learning? In M. Sharples (Ed.), Big Issuesin Mobile Learning: Report of a workshop by the Kaleidoscope Network of Excellence Mobile Learning Initiative (pp. 5-9). University of Nottingham. 
Alises Camacho, Ma Encarnación. Potencial pedagógico del Mobile Learning en el aula de música en secundaria

\section{AUTORA}

\section{Ma Encarnación Alises Camacho}

Diplomada en Magisterio por Ed. Musical por la Universidad de Burgos y funcionaria docente por esta especialidad en la Junta de Comunidades de Castilla La Mancha. Licenciada en Ciencias Religiosas por la Universidad de Comillas. Máster en Educación y Comunicación en Red por la UNED. Máster en Redes Sociales y Aprendizaje Digital por la UNED. Actualmente, Doctoranda en Educación en Entornos Digitales por la UNED y miembro del equipo pedagógico del Proyecto Crea Banda sonora de tu vida en Castilla La Mancha. 